\title{
1-D photonic crystals for photovoltaics
}

\author{
E.Gondek, ${ }^{* 1}$ P.Karasiński, ${ }^{2}$ \\ ${ }^{1}$ Institute of Physics, Cracow University of Technology, ul. Podchorążych 1, 30-084 Kraków, Poland, \\ ${ }^{2}$ Department of Optoelectronics, Silesian University of Technology, ul. B.Krzywoustego 2, 44-100 Gliwice, Poland
}

Received May 18, 2012; accepted June 9, 2012; published June 30, 2012

\begin{abstract}
The paper presents a theoretical analysis of photonic structures with 1-D photonic crystals on both sides of the slab glass and the results of experimental research. Within the scope of the theoretical analysis, using the $2 \times 2$ transfer matrix method, we have determined spectral reflectivity and transmittance characteristics. Consequently, we have determined the influence of the number of bilayers on maximum reflectivity and width of the main reflectivity peak. For the structure with four bilayers $\left(\mathrm{SiO}_{2} / \mathrm{TiO}_{2}\right)$ we have obtained a maximum reflectivity of 0.933 for the wavelength $\lambda_{r}=505 \mathrm{~nm}$ and full width at a half maximum of the main reflectivity peak of $F W H M=185 \mathrm{~nm}$.
\end{abstract}

Photovoltaic systems are being developed on the basis of nonorganis [1, 2] and organic [3-6] semiconductors. The essential part of the photovoltaic system cost is incurred by a photocell. Radical cost reduction of an electric power unit produced, can be achieved by applying solar radiation concentrators $[7,8]$. For the application in photovoltaics it is advisable to apply such concentrators focusing only this part of solar radiation spectrum on the photovoltaic structure which is effectively converted into electrical energy. Such requirements are satisfied by luminescence concentrators [9] and reflection concentrators with photonic mirrors [10]. The present paper involves onedimensional (1-D) reflective photonic structures deposited on glass substrates. A 1-D photonic structure (Fig. 1a), also called photonic crystal (PC) [11], is a multilayer stack in which the refractive index and layer thickness change periodically. The optical thicknesses of layers determine the resonance reflection wavelength $\lambda_{r}=d_{j} n_{j}$. In the paper we present the results of our theoretical analysis and experimental research of photonic structures with a PC on both sides of slab glass. We consider only PC structures with an even number $N$ of layers.

Figure $1 \mathrm{~b}$ presents the symmetrical optical structure with PCs present on both sides of glass substrate GS (structure $(\mathrm{HL})^{P} / \mathrm{GS} /(\mathrm{LH})^{P}, P=N / 2$, with notations as in Fig.1a). Such symmetrical structures can be fabricated using a dip-coating method. Glass substrates applied in photovoltaics have, most frequently, the thickness of $\sim 1 \mathrm{~mm}$. Therefore, there is no intereference between light beams which were reflected from the opposite photonic crystals. It means that reflectivity $R$ and transmittance $T$

\footnotetext{
*E-mail: gondek69@gmail.com
}

for the structure presented in Fig.1b can be determined by analyzing the intensity of successive reflected and transmitted beams.

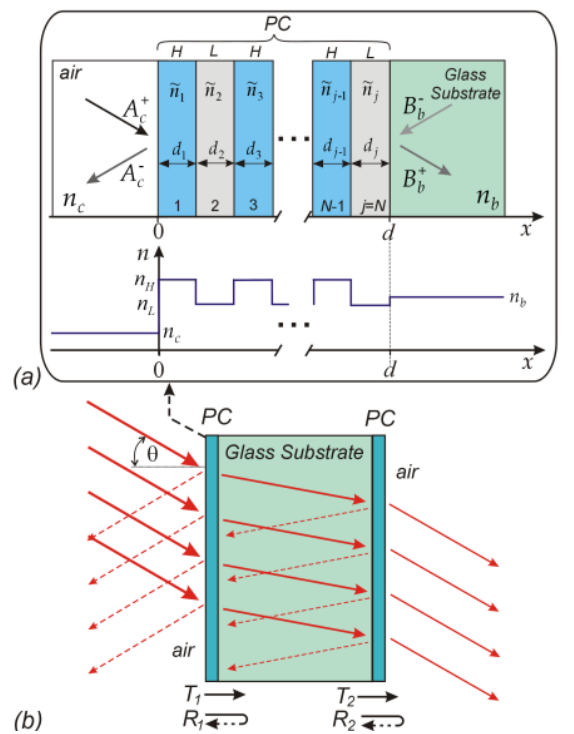

Fig.1. Optical structure with two photonic crystals (a), Photonic crystal with refractive index distribution (b).

We assumed in the analysis that the light transmitted in the substrate is attenuated. After each passage through the glass substrate, the intensity of light is reduced by a factor of $\gamma_{b}=\exp \left(-\mu_{b} x_{\theta}\right)$, where $x_{\theta}$ is the distance travelled by light from one side of the substrate to the other. Whereas $\mu_{b}=-$ $\ln \left(1-A_{b}\right) / d_{G S}$ is the absorption coefficient, where $d_{G S}$ is the glass substrate thickness and $A_{b}=1-T_{b}-R_{b}$ is the absorption of glass substrate, calculated from reflectivity and transmittance, determined experimentally.

The reflectivity and transmittance for the $\mathrm{PC} / \mathrm{SG} / \mathrm{PC}$ structure (Fig. 1b) are as follows:

$$
\begin{gathered}
R=R_{1}+\frac{\gamma_{b}^{2} T_{1} R_{2} T_{2}}{1-\gamma_{b}^{2} R_{2}^{2}}, \\
T=\frac{\gamma_{b} T_{1} T_{2}}{1-\gamma_{b}^{2} R_{2}^{2}} .
\end{gathered}
$$

Reflectivities $R_{1}, R_{2}$ and transmittances, $T_{1}, T_{2}$ (Fig. 1b) are obtained from the analysis of a single 1-D PC 
structure. In the theoretical analysis of PC structures we applied the transfer matrix $2 \times 2$ method [12]. We assumed that the materials of the layers are non-magnetic, isotropic, homogeneous and their refractive indices are complex $\tilde{n}_{j}=n_{j}+i \kappa_{j}$.

For the investigated multilayer structure (Fig. 1a), we can write as follows [12]:

$$
\left[\begin{array}{c}
A_{c}^{+} \\
A_{c}^{-}
\end{array}\right]=M\left[\begin{array}{l}
B_{b}^{+} \\
B_{b}^{-}
\end{array}\right]
$$

where $A_{c}^{+}$and $A_{c}^{-}$are the amplitudes of waves, the falling and reflected, respectively, on the left-hand side of the structure (Fig. 1a). Similarly, $B_{b}^{+}$and $B_{b}^{-}$are the amplitudes of respective waves in the glass substrate. Whereas $M$ is a transfer matrix of the multiplayer stack, defined as:

$$
M=\left[\begin{array}{ll}
M_{11} & M_{12} \\
M_{21} & M_{22}
\end{array}\right]=L_{c}^{-1}\left(\prod_{j=1}^{N} W_{j}\right) L_{b},
$$

$$
\text { where } \quad L_{\xi}=\left[\begin{array}{cc}
\left(n_{\xi}\right)^{\rho} & \left(n_{\xi}\right)^{\rho} \\
i \frac{k_{\xi, x}}{\left(n_{\xi}\right)^{\rho}} & -i \frac{k_{\xi, x}}{\left(n_{\xi}\right)^{\rho}}
\end{array}\right], \quad(\xi=c, b)
$$

are the interface matrixes, and:

$$
W_{j}=\left[\begin{array}{cc}
\cos \left(\tilde{k}_{j x} d_{j}\right) & -\frac{\left(\tilde{n}_{j}\right)^{2 \rho}}{\tilde{k}_{j x}} \sin \left(\tilde{k}_{j x} d_{j}\right) \\
\tilde{k}_{j x} \sin \left(\tilde{k}_{j x} d_{j}\right) & \cos \left(\tilde{k}_{j x} d_{j}\right)
\end{array}\right],
$$

where $\quad \tilde{k}_{j x}=k_{0} \sqrt{\tilde{n}_{j}-N^{2}}, N=n_{c} \sin \theta, \quad \rho=0 \quad$ for $s$ polarization and $\rho=1$ for $p$ polarization of incident light. Reflectivity and transmittance are respectively equal to: $R_{1}=\left|M_{21} / M_{11}\right|^{2}$ and $T_{1}=\left|1 / M_{11}\right|^{2}$. Making use of the calculation procedure presented above, we calculated total reflectivity $R$ and transmittance $T$ of the investigated structures. The calculations were carried out for perpendicular $(\theta=0)$ and oblique light incidence. In the calculations we applied refractive indexes of the layers $\left(n_{L}, n_{H}, n_{b}\right.$, denotations as in Fig. 1a), extinction coefficients $\left(\kappa_{L}, \kappa_{H}\right)$ and the absorption of glass substrate (soda-lime, Mentzel-Glaser) measured in an ellipsometric and spectrophotometric way, respectively In the theoretically analyzed structures, soda-lime glass was considered as substrate, silica layers $\mathrm{SiO}_{2}$ were applied as layers of a low refractive index $\left(n_{L}\right)$ and titania layers $\mathrm{TiO}_{2}$ were used as layers of a high refractive index $\left(n_{H}\right)$. Silica and titania layers have been fabricated via the solgel method [13, 14]. The refractive indices of the analyzed photonic structure for the resonance wavelength $\lambda_{r}=500 \mathrm{~nm}, \quad$ are $\quad$ as follows, $n_{L}=1.4968$, $n_{H}=2.1505+i \cdot 0.0284, n_{b}=1.5214$, whereas film thicknesses equal $d_{H}=58.1 \mathrm{~nm}$ and $d_{L}=83.5 \mathrm{~nm}$.

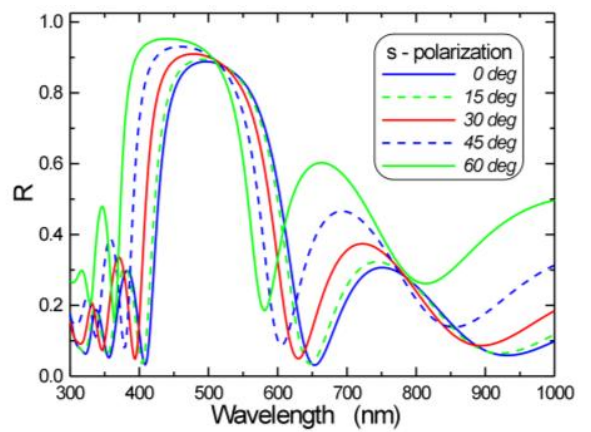

(a)

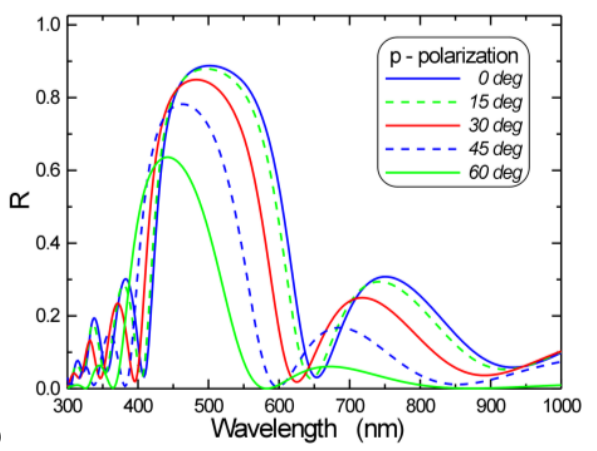

Fig. 2. Reflectances for selected angles of light incidence.

The characteristics of reflectances for selected angles of light incidence for polarizations $s$ and $p$, are presented in Fig.2a, and Fig.2b, respectively. In both cases we can observe a shift of reflectivity characteristics towards shorter waves with a rise of illumination angle $\theta$. This effect should be taken into account at the designing stage of a photovoltaic concentrator. In many other applications, it can be used also to modify the reflection spectrum of the actual photonic structure. For the $s$ polarization we can observe an increase in the reflectivity $R$ within the whole spectral range, and very small widening of the main peak with a rise in illumination angle $\theta$. And for the $p$-polarization, with a rise in illumination angle $\theta$ we can observe a decrease of reflectivity and the simultaneous decrease in the width of the main peak.

Figure 3 illustrates the influence of the number $P$ of bilayers in the PC structure on the height $R_{\max }$ of the main peak in the reflectivity characteristic and on FWHM. In the presented characteristics two change ranges can be 
observed. In the first range, when $P<6$ we can observe a strong increase of height $R_{\max }$ of the maximum reflectivity peak and a strong reduction of FWHM. In the second range when $P>6$ we can observe weak dependence of both height $R_{\max }$ of maximum reflectivity peak and of FWHM on number $P$ of bilayers.

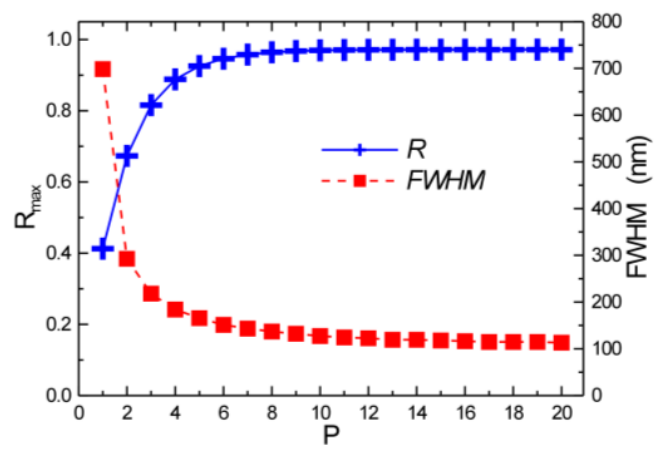

Fig.3. Influence of twolayers number on maximal reflectance and full width at a half maximum of the main reflectance peak.

Figure 4 presents spectral reflectivity and transmittance characteristics of the $(\mathrm{HL})^{4} / \mathrm{GS} /(\mathrm{LH})^{4}$ structure. Solid lines were used to plot experimental characteristics, of reflectivity $R$ and transmittance $T$, respectively. Dashed lines were used to plot theoretical characteristics calculated for the parameters of layers and substrate presented earlier. We can observe a good agreement between calculation results and experimental ones, both for reflectivity and transmittance characteristics. The differences between the calculated and measured characteristics are most probably caused by the difference between the refractive indices of component layers accepted for calculations and those of the fabricated photonic structure. The maximum height of the main reflectivity peak in the experimental characteristic is higher than in the calculation characteristic $\left(R_{c a l}=0.888\right)$. By approximating the measured reflectivity characteristic in the vicinity of the maximum with a $10^{\text {th }}$ degree polynominal, we determined the maximum value of refractivity $R_{\max }=0.933$ and its corresponding wavelength of $\lambda_{r}=505 \mathrm{~nm}$. The determined full width at a half maximum of the main reflectivity peak in the experimental characteristic $\left(F W H M_{\text {exp }}=210 \mathrm{~nm}\right)$ is also higher than in the case of the calculated characteristic $\left(F W H M_{c a l}=185 \mathrm{~nm}\right)$. The effect involving the widening of the main peak of the reflectivity characteristic has been caused by technological dispersion pertaining to the thickness of successive layers in the photonic structure.

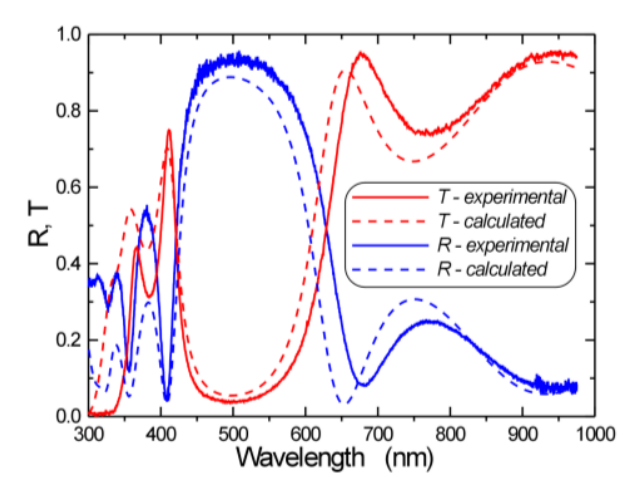

Fig. 4. Reflectances and transmittances for the structure of $(\mathrm{HL})^{4} / \mathrm{GS} /(\mathrm{LH})^{4}$.

In conclusion we can say that sol-gel processes and dipcoating technique are appropriate to the fabrication of 1-D photonic crystals with a large reflectance in the required spectrum range. Such structures can be applied to concentrate solar light in photovoltaic systems. The solgel technology is very efficient and. at the same time, it does not require expensive technological installations. It can be applied for mass production of high quality reflective elements for photovoltaics. When mesoporous titania layers of hydrophilic properties are applied as external layers, then such structures will have selfcleaning properties, which in view of photovoltaic applications is extremely important.

The work is sponsored by the National Science Centre within Grant 2011/03/B/ST7/0358.

\section{References}

[1] T.M.Razykov, C.S.Ferekides, D.Morel, E.Stefanakos, H.S.Ullal, H.M.Upadhyaya, Sol. Energy 85, 1580 (2011).

[2] B. Parida, S. Iniyanb, R. Goic, Ren. Sust. Energy Rev. 15, 1625 (2011).

[3] Organic electronics, edited by Hagen Klauk (Weinheim, WILEYVCH Verlag GmbH \& Co.KGaA, 2006)

[4] E. Gondek, I.V.Kityk, A.Danel, Mater. Chem. Phys. 112 (1), 301 (2008).

[5] E.Gondek, Y.Djaoued, J.Robichaud, P.Karasiński, I.V.Kityk, A.Danel, K.J.Plucinski, J. Mater. Sci.: Mater Electron, DOI 10.1007/s10854-012-0703-z.

[6] R.Signerski, G.Jarosz, Opto-Electron. Rev. 19, 468 (2011).

[7] A.G.Imenes, D.R.Mills, Sol. Energy Mater. Sol. Cells 84, 19 (2004).

[8] A.Zhedi, Ren. Sust. Energy Rev 15, 1609 (2011).

[9] B.C.Rowan, L.R.Wilson, B.S.Richards, IEEE J. Sel. Topics Q. Electron. 14, 1312 (2008).

[10] R.M.Almeida, L.M.Fortes, M.C.Gonçalves, Opt. Mater. 33, 1867 (2011).

[11] E. Yablonovitch, Phys. Rev. Lett. 58, 2059 (1987).

[12] G.V.Morozov, F.Placido, D.W.L.Sprung, J. Opt. 13, 035102 (2011).

[13] P.Karasiński, Opt. Appl. 36, 389 (2006).

[14] P.Karasiński, E.Gondek, S.Drewniak, I.V.Kityk, J. Sol Gel Sci. Techn. 61, 355 (2012). 